\title{
ASTROMETRY WITH THE LARGE SPACE TELESCOPE
}

\author{
W. F. VAN ALTENA \\ Yerkes Observatory, Williams Bay, Wis., U.S.A. \\ O. G. FRANZ \\ Lowell Observatory, Flagstaff, Ariz., U.S.A. \\ and \\ L. W. FREDRICK \\ Leander McCormick Observatory, Charlottesville, Va., U.S.A.
}

\begin{abstract}
The Large Space Telescope (LST), planned to be launched in 1980, will give an opportunity for astrometric observations of high quality to be secured.

A detailed survey is presented of the potentialities of the LST for studies of proper motions, parallaxes, star clusters, double stars, perturbed motion stars, occultations, fundamental astrometry and solar system observations.

The proposed characteristics and design capabilities of the LST are tabulated.
\end{abstract}

\section{Introduction}

In 1980 the National Aeronautics and Space Administration plans to launch into low earth orbit a diffraction limited 3-m telescope for astronomical observations. The Large Space Telescope (LST) will function as an observatory, and as such it will be equipped with several instruments, each designed for specialized observations. Current plans call for return of the LST to Earth by the Space Shuttle for servicing and possible replacement of instruments every two years and for a projected lifetime of 15-20 yr. Due to the very high spatial resolution, large scale and long projected lifetime, astrometry from the LST has been given a very high scientific priority since increases in precision over ground based astrometry by a factor of 10 are anticipated.

To insure that the LST and its instrumentation are designed to solve basic scientific problems, NASA has formed six Instrument Definition Teams composed of scientists and engineers who are charged with the responsibility for developing a preliminary design for the LST instruments. The Astrometry Team consists of W. F. van Altena as Team Leader, O. G. Franz, L. W. Fredrick, and a scientist and two engineers from NASA. NASA has set up a formal communications channel for each team through its liaison NASA scientist so that astronomers wishing to make suggestions to the Team can do so easily. The astrometry liaison scientist is Dr David B. Wood who is located at Goddard Space Flight Center in Greenbelt, Maryland.

The scientific potential to be gained through observations from the LST make astrometry a high priority area for all decisions effecting the LST. Depending on the instrumentation finally adopted, it will be possible, for example, to (1) improve the systematic accuracy of the FK4 system in some areas of the sky by a factor of ten or more and tie it into an absolute system of radio galaxies and quasars, (2) mea- 
sure parallaxes and proper motions ten times more accurately than presently possible thus firmly establishing the galactic distance scale and luminosity calibration for the luminous and some peculiar stars, (3) measure angular diameters for stars and the nuclei of galaxies for magnitudes brighter than 11-12, and (4) determine individual masses through 'visual' observations from the LST for about 100 spectroscopic binaries which will enormously improve the mass-luminosity relationship for the very massive stars. These and other areas of potential research are discussed in more detail in the following sections and two tables are given which summarize the basic characteristics of the LST and the capabilities required for the LST if astrometry is to be done from the LST.

\section{Parallaxes}

Parallax investigations are fundamental to astronomy. All distance determinations ultimately come back to parallaxes determined in the classical sense. In addition, the determination of stellar masses rests heavily upon parallax studies. Therefore, any instrument that promises higher accuracy in parallax determinations should be used and the LST is such an instrument.

LST parallax studies will break down into five areas of study: (1) parallaxes of faint stars (mag. 16 to 19); (2) parallaxes of stars in clusters used for photometric and distance scale calibrations (Hyades, etc.); (3) parallaxes of distant but important objects (planetary nebulae, etc.); (4) parallaxes of sub-dwarfs; and (5) parallaxes of certain key stars for calibration purposes.

Using the best techniques available at the present time, it is possible to determine the relative position of an average well-exposed stellar image on a photographic plate with an accuracy of \pm 0 ".02 (s.e.). This figure includes errors due to the limited spatial stability of the photographic emulsion, inaccuracies in defining the center of the photographic image, and possibly other instrumental effects. The pointing stability of the LST is projected to be $\pm 0.005 \mathrm{rms}$, therefore a short integration time, or

TABLE I

LST proposed characteristics

\begin{tabular}{|c|c|c|}
\hline \multirow{4}{*}{\multicolumn{2}{|c|}{$\begin{array}{l}\text { Primary mirror } \\
\text { Telescope length and diameter } \\
\text { Slew rate } \\
\text { Pointing stability }\end{array}$}} & $3-\mathrm{m} f / 2.2$ CerVit \\
\hline & & $12.7 \mathrm{~m}, 3.7 \mathrm{~m}$ \\
\hline & & $60^{\circ}$ in $40 \mathrm{~min}$ \\
\hline & & \pm 0.005 (s.e.) \\
\hline \multirow{3}{*}{$\begin{array}{c}\text { Cameras } \\
f \text {-ratio } \\
\text { Scale }\end{array}$} & \multirow{2}{*}{$\begin{array}{l}\text { Wide field } \\
f / 12\end{array}$} & High resolution \\
\hline & & $f / 96$ \\
\hline & $5.7 \mathrm{~mm}^{-1}$ & $0.7 \mathrm{~mm}^{-1}$ \\
\hline Field of view & $4: 8$ & $36^{\prime \prime}$ \\
\hline $\begin{array}{l}\text { Resolution } \\
(\lambda=3000 \AA)\end{array}$ & 0.17 & 0.03 \\
\hline $\begin{array}{l}\text { Limiting mag. } \\
(S / N=2,16 \mathrm{~min})\end{array}$ & 29 & 27 \\
\hline Detectors & \multicolumn{2}{|c|}{ 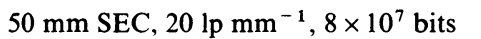 } \\
\hline
\end{tabular}


equivalently, five consecutive position measurements should yield a mean position of \pm 0 ".002 (s.e.). With an individual relative position determined ten times more precisely than is presently possible from Earth-based telescopes it should then be possible to determine parallaxes ten times more accurately or with an accuracy of \pm 0 ".001 (s.e.).

This high precision with the LST will yield parallaxes of objects at a distance of $40 \mathrm{pc}$ to $4 \%$ while they are now good to only about $20 \%$. Also, parallaxes at distances of $100 \mathrm{pc}$ will be good to $10 \%$. Thus stars used for calibrating photometric indicies, etc., will have much of the accidental scatter reduced, which will result in greatly improved calibrations. Since the Hyades cluster is so important to the cosmic distance scale it should receive high priority.

Many faint stars having large proper motions have been discovered by Giclas, Luyten, and others. These are nearby stars, but many are too faint for current earth based programs. A carefully selected sample of these stars requires parallaxes to add to our knowledge of the total mass in the solar neighborhood and the luminosity of the faintest stars.

Accurate parallaxes for the metal deficient Population II sub-dwarfs will yield their absolute magnitudes which in turn will reveal the distance scale for Population II stars and possibly shed some light on the ratio of hydrogen to helium in the very old stars. The presently available parallaxes are too inaccurate to solve these problems.

Finally, there are unusual objects, for example, the planetary nebulae, for which distances good to $10 \%$ will yield a wealth of knowledge and solve some long standing problems. The absolute magnitudes of the hot, degenerate, central stars of planetary nebulae are not known, hence the distances and masses of the nebulae are very uncertain. The confusion is so great that one indirect calibration method puts certain nebulae closer than others while another method has them just the other way around.

To accomplish the measurements of parallaxes we will need at least a five minute of arc field, unless high precision gyroscopes are used in the LST. Constraints upon the telescope and its systems depend upon the instrument used. Measurements at the focal plane equivalent to an accuracy of 0"003 are required.

\section{Astrometric Studies of Star Groups and Clusters with the LST}

The astrometric investigation of groups and clusters of stars with ground based telescopes has, of necessity, been almost completely limited to efforts of deciding which stars in the field of a group or cluster are likely to be members of the aggregate, and to a few investigations of possible overall expansion of such systems.

This limitation has been imposed by the accuracy with which proper motions can be determined from ground-based observations by combining recent measures with those on already existing first-epoch photographic plates obtained at most 60-70 years ago. Moreover, such investigations have been usually limited to stars brighter than the 13th visual mag., a limit dictated by the useful limiting magnitude on early 
epoch plates. Even if plates were now obtained to much fainter magnitudes, many decades would have to pass until proper motions of an accuracy useful even for membership investigations could be determined.

The standard error of a proper motion determined from one pair of plates with an epoch difference of 50 years is usually at least on the order of \pm 0 ."001, sufficient, in many instances, for membership studies, but inadequate to study the many interesting and important problems related to internal motions in groups and clusters.

The LST, on the other hand, can be expected to yield, over 5 yr periods, proper motions of stars in clusters and groups not only to magnitudes far beyond the reach of ground based instruments, but with an accuracy that will equal, if not exceed that obtained from ground based observations spanning 50 or more years. If such LST observations can be extended over periods of 10 or even $20 \mathrm{yr},-$ and most astrometry, even with the LST, will remain long range work - then proper motion determinations with standard errors of perhaps \pm 0 ".0005 or even better may be expected to be achieved. It is therefore proposed that LST-borne equipment be used for the following proper motion studies:

(1) To determine membership in selected open star clusters to the intrinsic magnitude limit in these clusters. Such a search for faint and the faintest members, in conjunction with appropriate photometric investigations, would not only help to establish the true and complete color-magnitude diagram and the luminosity function of these clusters, but should also result in the detection of the white dwarf members which should be present but, in most instances, have not been found in numbers predicted by evolutionary theory.

(2) To investigate, in clusters of various ages, sizes, and densities, the motions of samples of cluster members of various brightnesses and locations in these clusters, in order to study the character and distribution of their relative (internal) motions. Such studies are of great importance in conjunction with current ideas on the kinematics and the dynamical evolution of star clusters. They should provide answers to such questions as whether or not a Maxwell-Boltzmann velocity distribution has been established, indicating that some stars should have reached escape velocity from the cluster; whether dynamic sorting of stars according to their masses is present; whether distributions of internal motions might reflect star formation at several discrete epochs, etc. The questions are of the utmost importance in conjunction with efforts to understand the formation and the evolution of star clusters, and thus perhaps of stars in general.

\section{Objectives of Double Star Research with the LST}

Probably the most important purpose of double star research is that of direct determination of stellar masses. The knowledge of stellar masses is basic to our understanding of the internal structure and the evolution of stars; in combination with determinations of absolute bolometric magnitudes, it provides knowledge concerning the empirical mass-luminosity relation, a relationship of fundamental importance 


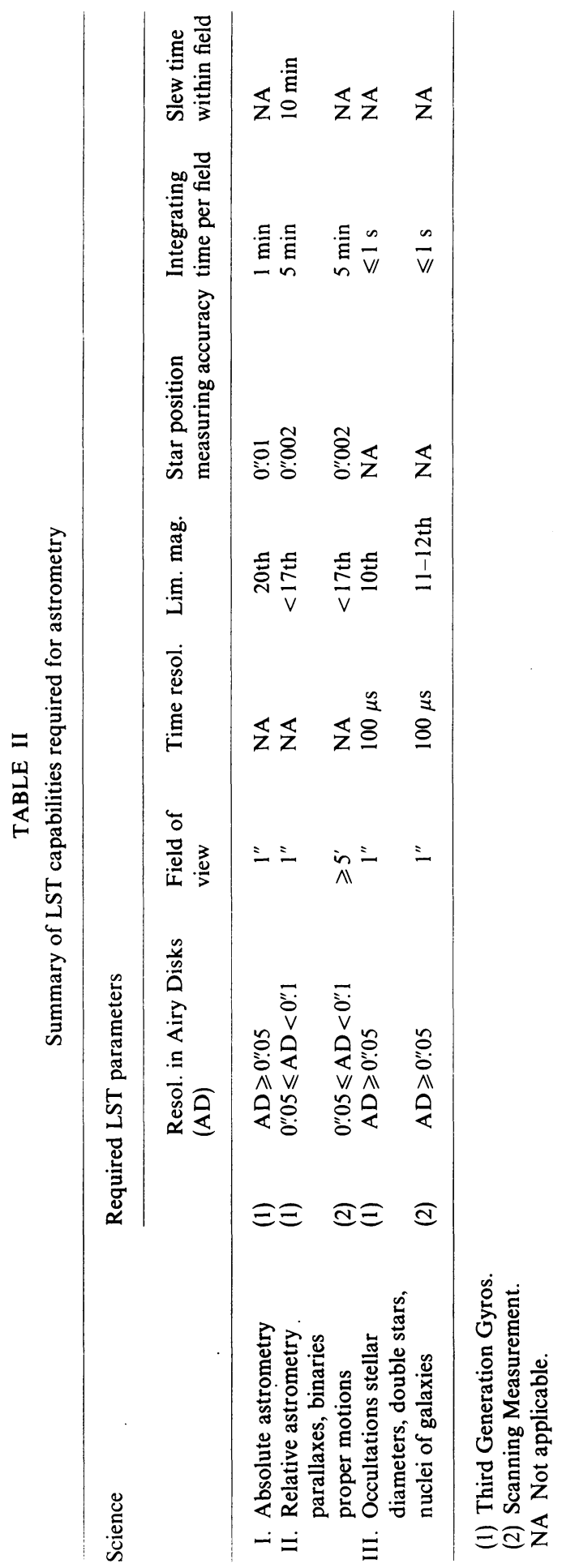


in astrophysics. It is particularly important to push our knowledge of the masses of stars to the limits of the largest and particularly of the smallest stellar masses.

Another major objective of double star research stems from the ever growing evidence that a large number, if not a majority of all stars, exists not as single stars, but as the components of double and multiple systems. This high incidence of duplicity and multiplicity may well be intimately related to the processes of star formation in general. The fact that certain types of objects, such as $U$ Gem variables, metallic-line stars, etc. occur in double stars only, must be of significance. It is particularly intriguing to speculate whether 'single' stars, except perhaps for those ejected from stellar systems as a result of dynamic evolution, may not be those possessing planetary systems.

To increase, if not to complete our information on the true incidence of duplicty and multiplicity among stars and to expand our knowledge on the existence of 'unseen' companions and of components of very low mass is one of the most challenging problems of current double star research. Such an extension of our knowledge of the mass-function to extremely small masses may provide, through inference and extrapolation, important empirical clues concerning the existence and the frequency of other planetary systems.

The components of a binary are almost certainly of common origin, their initial chemical compositions were most likely identical and they have differed possibly only in their original masses. They can therefore provide valuable test cases for current theories of stellar evolution, particularly since their present masses can sometimes be determined or at least reliably estimated in many instances, and provided that their colors and magnitudes can be accurately and individually measured. However, in order to explore fully and exploit this important aspect of double star research, it is necessary to resolve - also photometrically - the very closest.pairs and to cover the full range of stellar masses and their combinations from the very largest, usually occuring in spectroscopic binaries optically unresolved and unresolvable by ground based observations, to the very smallest whose existence is mostly inferred from perturbation analyses, but largely unconfirmed and unconfirmable by direct observation from the ground.

For these problems to be resolved or even to be attacked with some assurence of success, it is essential that double star observations be carried out in wavelength regions, magnitude ranges and, most importantly, at angular resolutions beyond those obtainable from the ground with any instrument.

The LST, on the other hand, presents a unique opportunity to carry out observations essential for the satisfactory solution of these problems. It is therefore proposed that LST-borne instrumentation be used to carry out the following programs:

(1) A specific search for all 'unseen' companions whose existence has been inferred from analyses of observed perturbations in the motions of nearby stars. Well over a dozen such perturbations, some with amplitudes of only a few hundredths of an arc second, are now known largely through the work of van de Kamp and his associates and many more will undoubtedly be detected. 
The angular separation of the 'unseen' companion from the visible star depends, of course, on the mass ratio between the objects. The masses inferred from perturbation analyses range from about one solar mass to values approaching that of Jupiter's mass.

Mere detection of 'unseen' components at their predicted positions with the LST would, of course, be an outstanding observational accomplishment, since it would provide direct confirmation of the results of determinations and interpretation of stellar perturbations often comparable in amplitude to the errors of observation. Actual measurement of photometric parameters and of a single orbital position of any 'unseen' component, on the other hand, would lead directly to the determination of its mass and luminosity free of the ambiguities often affecting the interpretation of perturbation measurements. Such determinations of the very smallest (stellar) masses would be of the utmost significance. This program should have highest priority among double star projects for the LST.

(2) Of nearly equal importance, however, is a searach for duplicty and multiplicity in nearby stars. The objects to be investigated in this manner may be selected from Gliese's Catalogue of Nearby Stars. This search, which should be extended to the infrared to facilitate the detection of faint, cool stellar components, can be expected to contribute significantly to our knowledge on the incidence of binaries and multiples whose components are too faint and too close to be detected by observations from the ground. Once detected, the binaries and multiples, many of which will have rapid orbital motions, should be observed by the LST astrometrically with a frequency and for a duration sufficient to determine their orbits and their masses and thus to provide data for defining the low-mass region of the empirical mass-luminosity relation. It is probable that most of the search for duplicity in the nearby stars can be carried out as a byproduct of occultation studies.

(3) Ground based investigations of spectroscopic binaries contribute to our knowledge on stellar masses only in the case of double-lined spectroscopic binaries which are also eclipsing variables. Observation of non-eclipsing, single-lined spectroscopic binaries with the LST, on the other hand, can be expected to resolve directly many of these objects and permit determination of their 'visual' orbits. Such orbit determinations would, in combination with known spectroscopic orbital elements, yield directly the masses for the components of many spectoscopic binaries and thus contribute importantly to our knowledge on the masses and the massluminosity relationship particularly for very massive stars.

(4) A search for duplicity and multiplicity among representative samples of many types of stars other than 'nearby' stars would further enhance our knowledge of the incidence of these phenomena among stars in general. Also, known binaries of separations too close to allow separate photometry of their components from the ground, should be observed with the LST to add to our knowledge on the physical properties of double star components, particularly in the case of pairs of special astrophysical interest. 


\section{Lunar Occultation Measurements of Stellar Diameters}

The knowledge of the diameter of a star is a topic of basic astrophysical interest, and in combination with other data the fundamental physical properties of the star can be determined. Except for the Sun, linear diameters of stars can be determined directly only for eclipsing spectroscopic binaries when the appropriate parameters can be measured. Angular diameters can be measured by several techniques, and if a parallax value is available, a linear diameter can be determined. The methods include the intensity interferometer, the Michelson interferometer, speckle interferometry, and lunar occultations, all of which encounter difficulties from brightness and suitability of available objects. It is difficult to evaluate the development of each of these techniques over the next 10 years, but it seems likely that improvements in the interferometric techniques may provide a considerable increase in the number of observed angular diameters. Nevertheless, lunar occultation results may be the best way of obtaining stellar diameters, at least for later-type stars.

Occultation observations could be useful for double star work in at least two ways. Although a single observation yields only a projected separation and magnitude difference, this information will be sufficient for a study of the frequency of duplicity in stars. Thus, as a byproduct of the occultation diameter measurements, we get statistics on the frequency of occurrence of double and multiple star systems. Also, occultation observations of that class of objects for which duplicity has been invoked to explain their unusual behavior would be a crucial test of that hypothesis. In addition, it may also be possible to measure the angular diameters of the nuclei of Seyfert or $N$-type galaxies in some cases through lunar occultations.

The principal advantage of spacecraft observation of a lunar occultation is the freedom from scintillation noise and atmospherically scattered moonlight, which constitute the major sources of noise in Earth-bound observations. The non-atmospheric component of the noise is the stochastic noise due to the number of detected photons in the sampling interval, but the characteristics of stochastic noise aré well understood and predictable. Other advantages are the lack of weather in space (which reduces the number of Earth-based observations by a factor of three) and the larger number of events which can be observed (due to the larger area covered and uncovered by the Moon as seen from a fast-moving satellite). In addition, the lunar motion across the star field as seen from the satellite is complex, and multiple occultations are possible near the altitude where the apparent lunar motion becomes very slow and finally is retrograde. Multiple observations at different contact angles could remove ambiguities caused by irregularities in the lunar surface. In cases where only one occultation is seen, observations in two or more wavelength regions can also resolve ambiguities caused by lunar limb distortions. Supplementary Earth-based observations would also be of value in this respect (in cases where the occultation could be seen on earth). Ultraviolet observations, which are most useful for early-type stars, should be possible, but due to the dependence of the fringe pattern on wavelength, smaller rocks $(15 \mathrm{~m}$ at $1500 \AA)$ could cause trouble (whereas the effective region of the lunar 
limb contributing to the occultation event for a point source at $6000 \AA$ is about $30 \mathrm{~m}$ ). However, spatial resolution is greater for shorter wavelengths.

The telescope velocity of the LST will be about 14 times greater than that of an Earth-based telescope at the point of central contact when the Moon's apparent velocity is greatest relative to the spacecraft. Due to this greater velocity, we will have proportionately more opportunities to observe occultations. The lunar motion at this point is about $3^{\prime \prime} \mathrm{s}^{-1}$, and the largest acceptable response time to record the fringe pattern is about $200 \mu \mathrm{s}$ per reading. In many cases, of course, the apparent lunar motion will be slower, and in a few special cases (near the retrograde motion) the occultation may last longer than is seen on Earth.

The time resolution required for lunar occultation studies could also be used for pulsar studies. In other words, the astrometric instrument to be used for occultations could probably also serve as a high speed photometer.

While the telescope aperture ( $3 \mathrm{~m}$ for the LST) imposes a lower limit on the detectable diameter of a star of about 0"0015 (due to destructive interference of fringes from opposite points on the mirror), this is not a serious problem. A $500 \AA$ optical bandwidth imposes a similar limit to the resulting measurement. It may be possible to place a mask of, say, $1 \mathrm{~m}$ in the perpendicular direction (to the path of the star across the lunar limb) across the center of the telescope aperture to reduce the fringe interference problem and thus allow smaller angular diameters to be detected. If the star is bright enough so that a narrow or intermediate band filter can be used, an additional resolution factor may be gained.

The problems caused by irregularities in the lunar limb will remain even with simultaneous observations in different wavelengths, multiple occultations when circumstances permit, and supplementary Earth-based observations. However, considering the relatively small amount of time required to observe an occultation, it seems that measurements of angular diameters of stars with the LST will provide much useful data with little additional effort in terms of instrumentation and design characteristics.

\section{Fundamental Astrometry from the LST}

Improvements in the fundamental system FK4 are possible with the LST and should be given high priority in the observing program. At the present time, our knowledge of Oort's constant $B$ of galactic rotation and of the distances of intermediate luminosity stars, through the use of secular parallaxes, is dependent on the FK 4 being an inertial reference system. Systematic errors in the FK4 result in similar errors in the derived quantities which can seriously affect our understanding of the structure and dynamics of the Galaxy. The FK4 and systems based upon it, such as the AGK3 and the Smithsonian Star Catalogue, are used for the determination of positions of solar system objects whose calculated orbits may be in error due to systematic errors in the FK4. These errors can seriously hamper projected observations of these objects from fly-by spacecraft or their later recovery after many years from Earth-based 
telescopes. Radio astrometry has progressed to a point where an intensive effort should be made to tie the radio absolute positions into the FK4 system, or vice versa, since the radio galaxies and quasars will certainly provide the best inertial reference system available. The LST has the potential to accurately accomplish this task because its large aperture will permit the observation of galaxies to the 20 th mag. while also allowing observations of the FK4 stars.

The potential of the LST for fundamental astrometry depends on the use of new high precision gyroscopes such as the Third Generation Gyros (TGG). The mean error of the FK4 system is given as \pm 0 ".014 to 0 ".040 in declination and \pm 0.015 to 0 ". 135 in right ascension, where the highest systematic accuracy is obtained near the equator and the lowest near the south pole. However, recent fundamental observations indicate that the errors may be several times larger in the southern hemisphere than estimated in the FK4. The projected accuracy of the TGG is \pm 0 ".04 (m.e.) over angles of $10^{\circ}$ and \pm 0 " 15 over angles of $60^{\circ}$ for a single measurement with a potential limiting accuracy approximately ten times better through repeated measurement. It seems likely that through repeated measurement and through the use of a network of observations over the sky it will be possible to achieve a significant improvement over the present fundamental system, perhaps by a factor of 10 or more in some areas of the sky.

It may be possible to obtain most of the fundamental observations needed for improvement of the FK4 through the necessary asquisition of guide stars for spectroscopic, photometric, etc. observations. The careful, rather than random, selection of guide stars will yield simultaneously highly accurate relative positions for the two guide stars and the object being measured and also the accurate inertial positions of those three stars.

\section{Solar System Observations}

In general, Earth-based solar system observations are hampered by insufficient resolution and limited by seeing conditions and scattered light. The LST provides' an immediate solution to many problems arising from insufficient resolution. Currently, for instance, the diameter of Pluto is poorly known. Greatly improved observations of the diameters of asteroids and of Pluto could be made with the LST. The mirror coatings in the LST should be smooth enough so that scattered light will not be a serious problem. Low scattered light levels would allow observations to determine, for example, the outward extent and detailed structure of the rings of Saturn.

The LST could also be useful in following interesting comets to large heliocentric distances. The tracking could be accomplished by taking observations of the comet on two successive satellite orbits and digitally subtracting them on board to determine the comets motion.

\section{DISCUSSION}

Mulholland: The SEC vidicon used in Mariner-type spacecraft is quite inadequate for astrometric purposes, due to instabilities and distortions. One would hope that one of the newer but much better imaging 
systems would be used. The alleged reliability of the vidicon is no advantage if the data are useless.

The utility of LST for lunar occultations may be marginal compared to smaller Earth-bound telescopes, because the time of an event will be sensibly different from one side of the mirror to the other. This will cause the interference fringes to be smeared in a way that may degrade the information content, particularly in the determination of stellar diameters.

Moffet: Why should you use the LST for occultation observations? These can be done just as well and infinitely more cheaply from the Earth's surface. Use the LST only for those things which cannot possibly be done from the Earth's surface.

Van Altena: Earth based occultation studies are limited by the noise introduced by the atmosphere. Gains can be made by observing from the LST. It should be kept in mind that an occultation requires only about one second of observing time to obtain very significant and valuable stellar angular diameters, and information on duplicity and multiplicity of stars.

Kovalevsky: Could the author explain how he intends to improve the FK4 system as it is now by a factor of 5 to 10 with angular observations good to 0.1 ?

Van Altena: The potential pointing accuracy is one part in $10^{6}$ of the angle traversed, which yields an accuracy of about $\pm 0^{\prime \prime} 04$ for a $10^{\circ}$ angle and $\pm 0^{\prime \prime}$. 15 for a $60^{\circ}$ angle. Repeated measurements should reduce the error to a value substantially below the systematic errors present in the FK4 which are as large as 0.8 in the southern hemisphere.

Eichhorn: There is another technique for determining accurately large angles between stars, possibly with an accuracy corresponding to a standard error in the order of 0.1 or so, which was investigated by Carol Williams at Tampa. It consists in topocentric observations of artificial satellites against a stellar background on photographic plates. There is a great deal of material already available to carry out this project in the satellite plates which have been taken so far.

This is not to say that the LST should not be used for the improvement of the absolute angular distances between the stars, especially since this is - as Strand pointed out - an unavoidable by-product of all the other work carried out on it.

Wood: Relevant to the valuable suggested use of the LST for obtaining data on binary stars it may be worth mentioning that the University of Sydney (Prof. Hanbury Brown) is at present seeking funds to build a large intensity interferometer which will be devoted partly to observation of binary stars to obtain accurate data including parallaxes.

Vasilevskis: Since the LST will not be an astrometric telescope, its share for astrometry is expected to be limited. Therefore, priorities will need to be assigned to various programmes. I think that the highest priority should go to close binaries.

Strand: I believe the programme of improving star positions with the gyros is the only astrometric programme which might have a chance to be included in the LST, because of conflict with many far more sophisticated astrophysical programmes.

Fricke: Although the positional accuracy which can be reached with the space telescope is not exceedingly high compared with modern fundamental methods, the advantages are considerable for faint stars. 